\title{
The Application of Morphological Analysis on the Experimental Teaching Assessment of Creative Problem Solving
}

\author{
Tsui-Lien Shen and Jiin-Chyuan Lai
}

\begin{abstract}
More innovative imagination and concrete results would be meaningfully produced to become civilized products and cultural creativity if the creative techniques can be employed through creative problem-solving teaching. The purpose of this study was to apply the morphological analysis on experimental teaching. ANCOVA was employed to analyze the change of the students' creative problem-solving thinking, and qualitatively analyze the creative problem-solving process of the team's performance imaginary creative works. The participants of this study were 105 students from the University of Science and Technology, which were divided into experimental group and control group. The research method was quasi-experimental research with the Nonequivalent Pretest-Posttest Designs. The research instruments included the Abbreviated Torrance Test for Adults (ATTA), the creative problem-solving teaching design with learning perception questionnaire, and the expert assessment with creative imagination work scale. The conclusions of this study were as the followings: 1. In the creative problem-solving, the students in the experimental group achieved higher significant level in terms of producing innovative ideas than the students in the control group; whereas did not achieve the significant level regarding the awareness of challenges, preparing for actions and planning for methods. 2 . In the expert assessment with creative imagination work scale, the students in the experimental group achieved higher significant level in terms of relevance \& effectiveness, problem concentrating, elaboration, originality, multiple-possibilities, connectivity, transcendence than the students in the control group. 3. In the Qualitative analysis regarding creative problem-solving, the students were recognized lacking of practical experiences; it was difficult for them to conduct the preparation for actions and planning for methods to solve the practical problems.
\end{abstract}

Index Terms-Creative techniques, teaching and Assessment, morphological analysis, creativity, imagination.

\section{INTRODUCTION}

Creative Problem Solving (CPS) aims to create a more appropriate and original solution [1]. Meanwhile, CPS is the process of applying creative thinking to solve the problem through continuous practical verification to develop a creative problem-solving model that demonstrates the specific results of creative thinking with diversity, originality, effectiveness. The differences of creative problem solving and general problem solving are as the followings: 1).

Manuscript received November 6, 2017; revised March 13, 2018

Tsui-Lien Shen is with the Center for General Education, National Formosa University, Taiwan (e-mail: tlshen@nfu.edu.tw).

Jiin-Chyuan Lai is with the Department of Applied Foreign Languages, Transworld University, Taiwan (e-mail: marklai07@gmail.com).
Problem solving refers to the definition of a problem area that can be defined in a clear and organized manner; and CPS is a simulation of ambiguous problem solving and methods [2] [3]. 2). Problem solving needs to be addressed by memory and by well-known expertise, knowledge, and CPS needs to be addressed through creative thinking [4], [5]. 3). The desired outcome of the problem solving can be the result that already exists or can be obtained easily, and the problem-solving needs to concentrate on and seek knowledge extensively; and the results expected by CPS are not likely to exist but are currently available. The result of CPS is the rich imagination and creativity [6], [7].

As a result of CPS development with the variety of models, this study adopted Isaksen, Dorval, \& Treffinger [8] four constitutive elements of a creative problem-solving and eight steps: 1). Understanding the challenges: 1.1 constructing opportunities, 1.2 exploring data, 1.3 framing problems. 2). Generating ideas: 2.1 generating innovative ideas. 3). Preparing for action: 3.1 building acceptance, 3.2 developing solutions. 4). Planning your approach: 4.1 designing process, 4.2 appraising tasks as the basis for teaching design activities.

Morphological analysis concept is proposed by the astronomer F.Zwicky, it is a process for creating new ideas by analyzing the form and structure of existing ones and changing the relationships of their components. M.S. Allen published book" Morphological creativity" in1962 [9]. The type of analysis method applied rapidly to the fields of psychology, management, engineering, and computer technology. Especially in Japan, many product developers used the form of analysis to the concept of creative ideas, through the analysis of integrated, inductive interpretation and diffusion of convergence of thinking methods, product development design output with imagination creative products. Application of type analysis is as the product development and design of creative steps, including the 5 processes [10], [11].

1) Lists the independent elements.

2) Imagine variable parameters.

3) Design matrix.

4) Choose the best solution.

5) Formation of new ideas to specific creation.

This research is the application of the creative steps of the morphological creativity and integrated the prerequisite factors of the students, learning tasks, and teaching context of planning for the basis of teaching activities for students to learn the creative problems solving.

\section{RESEARCH METHODS}

\section{A. Research Design}


The quasi-experimental research was adopted with the Nonequivalent Pretest-Posttest Designs as Table I.

The participants were divided into experimental group and control group. The experimental group applied instructional design with CPS of morphological analysis as experimental interventions. The control group was instructional design for General. Both of these two groups accepted CPS Abbreviated Torrance Test for Adults (ATTA) and CPS of Teaching Design with Learning Perception Questionnaire as pretests. After 6 hours during 3 weeks by experimental interventions, the experimental group accepted ATTA, CPS of Teaching Design with Learning Perception Questionnaire, expert assessment of creative imagination work and self-assessment of CPS as posttests.

TABLE I: RESEARCH DESIGN

\begin{tabular}{lcll}
\hline \hline Groups & Pretest & Experimental & Posttest \\
\hline Experimental & 1. ATTA & Instructional & 1.ATTA \\
group & 2. CPS of & design with & 2. CPS of \\
& Instructional & CPS of & Teaching Design \\
& Design with & Morphological & with Learning \\
& Learning & Analysis & Perception \\
& Perception & & Questionnaire \\
& Questionnaire & & 3. Expert \\
& & & assessment of \\
& & Instructional & creative \\
Control group & & design for & imagination work \\
& & General CPS & 4.Self-assessment \\
& & & of CPS \\
\hline \hline
\end{tabular}

\section{B. Participants}

The students as subjects were randomly assigned by the computer into 4 classes. The research adopted purposive selection to choose one class with 53 students into the experimental group before conducting experiment, and the other class with 52 students in the control group.

\section{Instruments}

In this research, there were four types of instruments employed as followings:

1) Abbreviated Torrance Test for Adults (ATTA) in Chinese version: ATTA original was created by Goff., \&Torrance [12] and ATTA in Chinese Version by Chen [13]. The study of the retest reliability in the coefficient was .614.

2) In the CPS instructional design with learning perception questionnaire was constructed by 3 experts' suggestions who specialized in academic and practical fields among design, creativity and teaching, the coefficient of the Cronbach $\alpha$ level of internal consistency reliability was .981 .

3) In the expert assessment with scale of creative imagination work referenced researchers' scales of Cropley \& Cropley [14], Cropley \& Kaufman [15], Wang [16] and integrated into list of scale items. The internal consistency reliability coefficient of Cronbach $\alpha$ level was .882.

4) In the self-assessment of CPS referenced ideas of Isaksen, Dorval, \& Treffinger [8] and Treffinger, Selby, \&Isaksen [17] to make items of self-assessment of CPS. The internal consistency reliability coefficient of Cronbach $\alpha$ level was .991.

\section{Data Analysis}

The quantitative data received by the research instruments with eliminating the invalid samples were conducted by SPSS to process ANCOVA analysis with the pretest of Abbreviated Torrance Test for Adults (ATTA) and the CPS teaching design with learning perception questionnaire as covariance; and the expert assessment with creative imagination work scale, and self-assessment of creative problem-solving as the dependent variable; ANCOVA was conducted to test the differences in the experimental group and control group. The analysis of works was conducted on the basis of assessment sheet of performance to analyze the influential elements of creative problem solving.

\section{RESUlTS}

\section{A. Application of Morphological Analysis on the Creative Problem Solving Assessment by Creators' Team}

From Table II" ANCOVA in Self-assessment of Creative Problem-solving," both of experimental and control groups accepted experimental interventions and works' assessments simultaneously. To know what effects on application of morphological analysis on the creative problem solving this research design made assessments by creators' teams. It shows that the score received by the students in the experimental group $(F=10.238, p=.002)$, which was significantly higher than that of the control group. It indicated that the significance of promoting generating new ideas in CPS. The scores received in terms of understanding the challenge $(F=2.225, p=139)$, preparing for action $(F=2.589, p=.111)$, and planning your approach $(F=2.589$, $p=.111)$ indicated that there were no significant benefits achieved.

Lothane [18] argued that imagination was a type of ability to form an image by connecting matters with visualization. Generating new ideas are needed the bases of visualization experiences and inspired imagination. Moreover, imagination had a variety of possibilities, connectivity and boundary-crossing [16] to generate new ideas certainly requires psychological imagination, which can be achieved by collecting information and drawing processes and to visualize the images with producing more things to develop products.

\begin{tabular}{lllllll}
\multicolumn{1}{c}{ TABLE II: ANCOVA IN SELF-ASSESSMENT OF CREATIVE PROBLEM-SOLVING } \\
\hline \hline \multicolumn{1}{c}{ Level } & Group & $N$ & $\begin{array}{c}\text { Adjusted } \\
\text { Mean }\end{array}$ & $\begin{array}{c}\text { Standard } \\
\text { Error }\end{array}$ & $F$ & $p$ \\
\hline Understanding & Exp. & 14 & 4.914 & .231 & 2.225 & .139 \\
the challenge & Con. & 14 & 4.423 & .226 & & \\
Generating & Exp. & 14 & 8.623 & .364 & 10.238 & .002 \\
ideas & Con. & 14 & 6.962 & .357 & & \\
Preparing for & Exp. & 14 & 5.698 & .191 & 2.589 & .111 \\
action & Con. & 14 & 5.250 & .196 & & \\
Planning your & Exp. & 14 & 7.706 & .208 & .699 & .405 \\
approach & Con. & 14 & 7.959 & .213 & & \\
\hline$* p<.05 * * p<.01$ & $* * * p<001$ & & & & \\
\hline
\end{tabular}

\section{B. Application of Morphological Analysis on the Experts' Assessment with Creative Imagination Works}

From Table III "Application of Morphological Analysis on 
the expert assessment with creative imagination works," two experts' were invited to evaluate creative imagination works of creators' team after morphological teaching processes. There are 7 levels as different levels to distinguish imagination works. It includes relevance $\&$ validity, problem concentrating, elaboration, originality, multiple possibilities, connectivity, transcendence [14]-[16]. It shows that the score received by the students in the experimental group in terms of relevance and validity $(F=12.889, p=.001)$, problem concentrating $(F=12.202, p=.001)$, elaboration $(F=14.039, p$ $<.001)$, originality $(F=10.880, p=.001)$, multiple possibilities $(F=14.355, p<.001)$, connectivity $(F=9.660$, $p=.002)$, transcendence $(F=10.183, p=.002)$, total $(F=4.468$, $p=.037)$ had reached the significance.

It means that students in experimental group received in terms of relevance \& validity, problem concentrating, elaboration, originality, multiple possibilities, connectivity, transcendence and in total were higher than those the students in the control group did in the expert assessment with creative imagination works. The results indicated there was a great significance in terms of promotion of creative imagery works in this experimental teaching study.

TABLE III: ANCOVA ON EXPERTS' ASSESSMENT WITH CREATIVE IMAGINATION WORKS

\begin{tabular}{lllcccr}
\hline \hline \multicolumn{1}{c}{ Level } & Group & $N$ & $\begin{array}{c}\text { Adjusted } \\
\text { Mean }\end{array}$ & $\begin{array}{c}\text { Standard } \\
\text { Error }\end{array}$ & $F$ & $p$ \\
\hline relevance \& & Exp. & 14 & 23.962 & .860 & 12.889 & .001 \\
validity & Con. & 14 & 19.612 & .834 & & \\
problem & Exp. & 14 & 23.651 & .847 & 12.202 & .001 \\
concentrating & Con. & 14 & 19.483 & .822 & & \\
elaboration & Exp. & 14 & 22.703 & .823 & 14.039 & $<.00$ \\
& Con. & 14 & 18.357 & .799 & & 1 \\
originality & Exp. & 14 & 23.500 & .867 & 10.880 & .001 \\
& Con. & 14 & 19.472 & .841 & & \\
multiple & Exp. & 14 & 23.963 & .855 & 14.355 & $<.00$ \\
possibilities & Con. & 14 & 19.400 & .829 & & 1 \\
connectivity & Exp. & 14 & 22.409 & .827 & 9.660 & .002 \\
& Con. & 14 & 18.787 & .802 & & \\
transcendenc & Exp. & 14 & 21.749 & .824 & 10.183 & .002 \\
e & Con. & 14 & 18.044 & .799 & & \\
total & Exp. & 14 & 149.202 & 7.790 & 4.468 & .037 \\
& Con. & 14 & 125.389 & 7.875 & & \\
\hline \hline$* p<.05 * * p<.01 * * * p<001$ & & & &
\end{tabular}

\section{Selected Case Analysis}

This study shows that the creative problem solving in terms of generating ideas performed quite effective showed as Table IV. The researchers selected one case analysis of creating imagination lamp--gymnastics player with cat light as Fig. 1 after assessment of creative imagination works. The creators often used techniques of relevance \& validity, problem concentrating, elaboration, originality, multiple possibilities, connectivity, transcendence, and 5W2H (Who? What? Why? When? Where? How? How much?) as their creative thinking and design.

They applied to collect new ideas, thinking about the parameters and matrix configuration. To analyzed the thinking processes of Morphological Analysis as following,

1) Lists the independent elements: the creator cited objects, action, emotion, and utensils.

2) Imagine variable parameters: for example, objects were as imaginative as dog, cat, jellyfish, cockroach, and kite. The others are as Fig. 1.
3) Design matrix: there are 400 ideas by variable parameters $(5 \times 4 \times 5 \times 4=400)$.

4) Choose the best solution: there are three kinds of solution as followings, the creator deeply imagine how to make formations i) cat/dancing/ ambiguous/ribbon ii) cockroach/ attack/ attack/fan iii) kite/ rotate/ smile/plate. Finally, imaging one cat is dancing with ribbon in light of ambiguous situation is the best solution.

5) The formation of new ideas to specific creation: i) The cat is like a gymnastics player, ii) The cat is dancing with ribbon to move. iii) The cat is in an ambiguousin place. (iv) The ribbon is shining within LED-light.

It would help people to obtain the relevant information into the brain and to transform into a specific feasible solution and the creative problem solving can even more effective. However, comparing with the aspects of understanding challenges, preparing for action, design measures in creative problem solving, if the creator could devote more professional development and practices to produce more feasible and practical works. The creator would propose the formation of a community with generating new ideas, culture, and creative action would make practices of creative problem solving.

TABLE IV: CREATIVE PROBLEM SOLVING ON CREATING GYMNASTICS

\begin{tabular}{|c|c|c|c|}
\hline Objects & Action & Emotion & Utensils \\
\hline $\operatorname{dog}$ & run & smile & ribbon \\
\hline cat & attack & sad & flute \\
\hline jellyfish & rotate & angry & fan \\
\hline cockroach & dancing & pissed off & plate \\
\hline kite & & ambiguou & \\
\hline
\end{tabular}

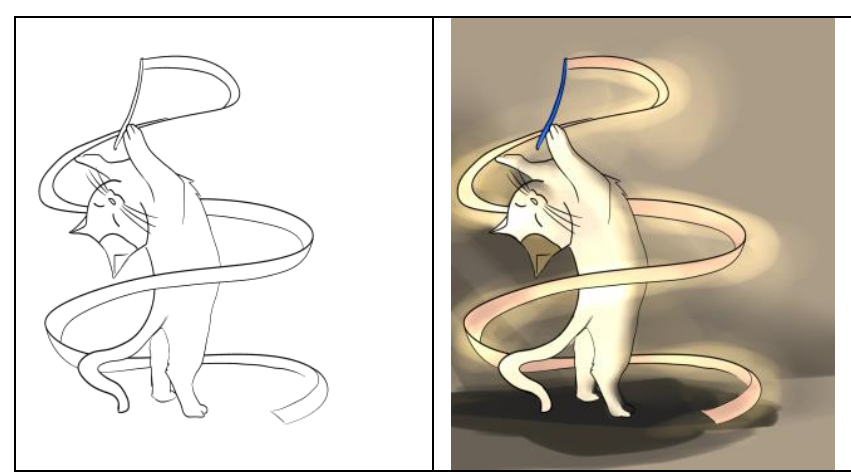

Fig. 1. Gymnastics player with cat light.

\section{CONCLUSIONS AND RECOMMENDATIONS}

Based on the application Morphological Analysis in the creative problem solving assessment, the only significant difference in the experimental group was in generating new ideas. There was no significant difference in understanding challenges, preparing for action, and planning your approach in the experimental and control groups. In the application of Morphological Analysis on the expert assessment with creative imagination works, the score received by the students in the experimental group in terms of the relevance and validity, problem concentrating, elaboration, originality, multiple possibilities, connectivity, transcendence, and total had higher scores than those in the control groups and reached the significance. In the application of Morphological Analysis on the creation of imagination works in a qualitative 
assessment, it indicated that this teaching experiment did have the influence on the imagination works regarding the relevance and validity, problem concentrating, elaboration, originality, multiple possibilities, connectivity, transcendence. The creators can produce the products only with putting into practice in reality in terms of understanding the challenges, preparation for action and planning your approach.

Creative Problem Solving (CPS) aims to create a more appropriate and original solution [1]. As Zhu, Nagalingam, \& Hsu [19] argued that creative problem solving can be more specifically conducted in meta-action by combining creative techniques with methodology and knowledge. Hence, CPS can be also interpreted as knowledgeable creative problem-solving methodology, KCPS. Therefore, this study of the application of Morphological Analysis demonstrated the positive influence on integrating teaching design to have students create imaginative works through creative problem solving. To explore the way how to process the teaching design and instruct the students to produce the imaginative and creative works should be essential for creative problem solving.

\section{REFERENCES}

[1] M. Boden, "Creativity and unpredictability," Stanford Humanities Review, vol. 4, 1995.

[2] D. K. Carson and M. A. Runco, "Creativity problem solving and problem finding in young adults: Interconnections with stress, hassles, and coping abilities," Journal of Creative Behavior, vol. 33, pp. 167-190, 1999.

[3] J. W. Getzels and M. Csikszentmihalyi, The Creative Vision: A Longitudinal Study of Problem Finding in Art, New York: JohnWiley \& Sons, 1976.

[4] D. C. Geary, "The origin of mind: Evolution of brain, cognition, and general intelligence," Washington, DC: American Psychological Association, 2005.

[5] M. J. Kirton, Adaption-Innovation in the Context of Diversity and Change, London: Routledge, 2003

[6] M. Beer and N. Nohria, Breaking the Code of Change, Boston: Harvard Business School Press, 2000.

[7] L. Bossidy and R. Charan, Execution: The Discipline of Getting Things Done, New York: Crown Business, 2002.

[8] S. G. Isaksen, K. B. Dorval and D. J. Treffinger, Creative Approaches to Problem Solving: A Framework for Change, Dubuque, Iowa: Kendall/Hunt, 2000.

[9] M. S. Allen, Morphological Creativity: The Miracle of Your Hidden Brain Power, Englewood Cliffs, New Jersey: Prentice-Hall, 1962.

[10] T. Ritchey, "Fritz Zwicky, morphologie and policy analysis," presented at the $16^{\text {th }}$ Euro Conference on Operational Analysis, Brussels, 1998.
[11] T. L. Shen, Creative Thinking, and Design, Taipei: Wu-Nan Publisher, 2017.

[12] E. P. Torrance and K. Goff, "A quiet revolution," Journal of Creative Behavior, vol. 23, pp.136-145, 1989.

[13] C. Y. Chen, Abbreviated Torrance Test for Adults (ATTA) in Chinese Version, Taipei: Psychology Publisher, 2006.

[14] D. Cropley and A. Cropley, "Cropley, recognizing and fostering creativity in technological design education," Int J Technol Des Educ, vol. 20, pp. 345-358, 2010.

[15] D. Cropley and J. C. Kaufman, "Measuring functional creativity: empirical validation of the creative solution diagnosis scale (CSDS)," Journal of Creative Behavior, vol. 46, pp. 119-137, 2012.

[16] H. H. Wang, "The cultivating and evluation of imagination in educational process," National Science Council Final Report of Project, NSC 98-2511-S-003-007-MY2, 2011.

[17] D. J. Treffinger, E. C. Selby, and S. G. Isaksen, "Understanding individual problem-solving style: A key to learning and applying creative problem solving," Learning and individual difference, vol.18, pp.390-401, 2008.

[18] Z. Lothane, "Imagination as reciprocal process and its role in the psychoanalytic situation," International Forum of Psychoanalysis, vol. 16, pp.152-163, 2007.

[19] L. Zhu, S. V. Nagalingam, and H. Y. Hsu, "Toward a creative problem-solving methodology with knowledge provision," Applied Artificial Intelligence: An International Journal, vol. 25, pp. 836-881, 2011.

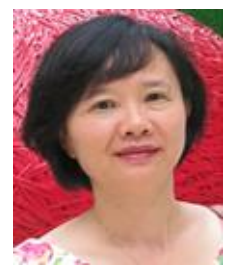

Shen Tsui-Lien is a professor at Center for General Education, National Formosa University, Taiwan, an was also ever a visiting scholar at University of Helsinki, Finland and FH Technikum Wien, University of Applied Science, Austria. Her research interests and specialties included: creativity in education, design thinking, curriculum and instruction, instruction design and teaching for creativity. She would like to create new ideas in teaching for students to develop creative/imaginative thinking and inspire students' learning motivation to put creative ideas into prototyping of works. The focus of her publications is on creativity covered in her books, papers, project reports, and patents.

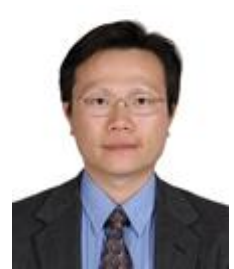

Jiin-Chyuan Mark Lai is a researcher concentrating in the area of interdisciplinary language and culture specifically in English and Chinese. Dr. Lai completed his doctoral dissertation, titled "Teaching Culture as Metaphor to Adult Learners in English as a Foreign Language Curriculum"; receiving his Ph.D. degree from University of Louisville, Kentucky, U.S.A. in May 2007. In 2003, while serving as an administrative assistant in the student life department at Spalding University, Kentucky, U.S.A, Dr. Lai received the award Presented to MARK LAI for Outstanding Service to Spalding University. Dr. Mark Lai is focusing his EFL research using mixed-methods on areas of language development and competence, linguistics, cross cultural communication, English-Chinese translation/interpretation, especially translation of culturally-laden language and English/American literature teaching. 